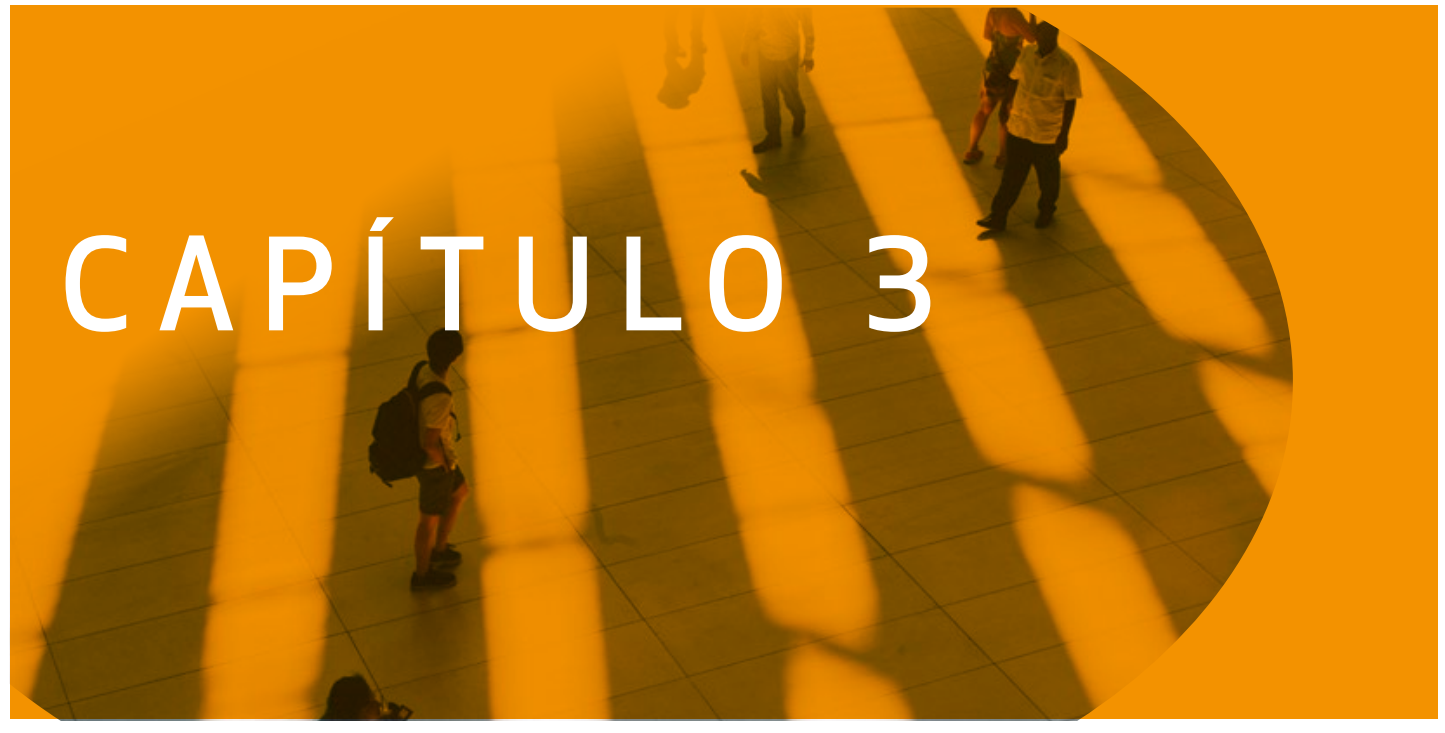





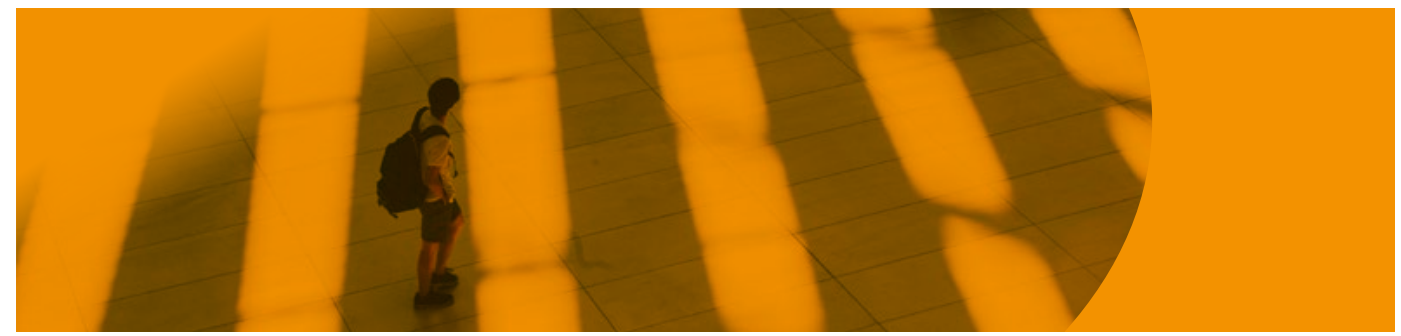

\section{Oportunidades para la educación contable en Colombia}

\section{Martha Lucía Fuertes Díaz}

Doctora en Administración Gerencial (C), Universidad Benito Juárez, México. Magíster en Administración con énfasis en Finanzas Corporativas, Universidad ICESI, Colombia. Especialista en Finanzas con concentración en Finanzas Avanzadas, Universidad ICESI, Colombia. Contadora Pública, Universidad Santiago de Cali, Colombia. Docente Universidad Nacional Abierta y a Distancia, UNAD, Colombia. Investigadora principal Grupo de Investigación ilama.

Correo electrónico: martha.fuertes@unad.edu.co

ORCID: http://orcid.org/0000-0001-8468-1867

Google Scholar: https://scholar.google.com.co/citations?user=|P5s-

\section{Marilú Avendaño}

Magíster en Educación, Instituto Tecnológico de Monterrey, México. Especialista en Gestión de Proyectos, UNAD, Colombia. Especialista en Educación Superior a Distancia, UNAD, Colombia. Administradora de Empresas, UNAD, Colombia. Docente e investigadora Grupo de Investigación GIEPE.

Correo electrónico: marilu.avendano@unad.edu.co

ORCID: https://orcid.org/0000-0001-7578-5816

Google Scholar: https://scholar.google.es/citations?user=_anOzf4AAAAJ\&hl=es 


\section{Introducción}

En el primer capítulo, del presente libro se identificaron las políticas públicas que regulan el desempeño de los profesionales de la contaduría pública y la educación contable superior. Se estableció la importancia del desarrollo de competencias disciplinares, la formación integral y el ejercicio ético como garantías para la sociedad y el sector empresarial, pues estas características le permiten convertirse en un verdadero garante y generador de la confianza pública como coadyuvador para el desarrollo económico de las organizaciones, la sociedad y el país.

En el segundo capítulo se establecieron las necesidades del tejido empresarial, desde los diferentes sectores de la economía que cubren las diferentes regiones de Colombia y las necesidades de la población respecto de la formación profesional del contador público. Estos son factores fundamentales que permiten impactar efectivamente la sociedad y su tejido empresarial.

En el presente capítulo se establecen las oportunidades para el sector educativo colombiano respecto de las propuestas de formación para los profesionales de la contaduría pública. Se toma como base el contexto de desarrollo de la educación contable en el ámbito nacional e internacional, las necesidades de la población y las organizaciones del país.

En los últimos años, la educación se ha constituido en base fundamental para orientar el desarrollo social y económico del país, como se apreció desde el Plan Nacional de Desarrollo 2014-2018 Todos por un nuevo país: Paz. Equidad, Educación, que orientó sus esfuerzos, entre otros, a una Colombia equitativa y sin pobreza extrema. Lo cual consagra en su objetivo 3:

[...] la educación es el más poderoso instrumento de igualdad social y crecimiento económico en el largo plazo, con una visión orientad a cerrar brechas en acceso y calidad al sistema educativo, entre individuos, grupos poblacionales y entre regiones, acercando al país a altos estándares internacionales y logrando la igualdad de oportunidades para todos los ciudadanos. 
A esta propuesta se articuló el Ministerio de Educación Nacional, que estableció líneas estratégicas para orientar la política educativa encaminada al logro de una "Colombia, la mejor educada en 2025". El objetivo es avanzar para ser el país más educado de América Latina, concentrar esfuerzos en la ampliación de cobertura, y mejorar la pertinencia de los programas de educación superior a partir de cinco pilares: excelencia docente, jornada única, Colombia bilingüe, Colombia libre de analfabetismo y más acceso a la educación superior de calidad. A nivel regional y local, en los últimos años se han articulado los respectivos planes de desarrollo de los departamentos y municipios del país, donde la educación es considerada generador de desarrollo y bienestar comunitario.

Además, es importante destacar el Acuerdo por lo Superior 2034 liderado por el Ministerio de Educación Nacional y el Consejo Nacional de Educación Superior CESU, el cual establece la propuesta de política pública para la excelencia de la educación superior en Colombia en el escenario de la paz, con la participación de la comunidad académica, el gobierno nacional y el sector productivo, sumando en total 33.000 participantes Con este acuerdo se busca avanzar en el logro de:

- Educación inclusiva, con acceso, permanencia y graduación para todos.

- Educación superior reconocida por su alta calidad.

- Investigación que responda mejor a las necesidades locales.

- Mejores condiciones para el desarrollo de las comunidades académicas.

- Educación superior universal y de calidad en todo tiempo y lugar, gracias a las tecnologías de la información.

- Educación superior con un enfoque internacional.

- Covertura de educación superior en todas las regiones.

- Facilitar la movilidad de estudiantes entre las instituciones educativas y sus diversos niveles.

- Asegurar la consolidación de la estructura y el gobierno del sistema de educación superior.

- Sostenibilidad financiera para todas las instituciones de educación superior. 
Desde los diversos análisis y estudios realizados, tanto en el contexto nacional como mundial, respecto de la profesión del contador público, las necesidades de las organizaciones, la sociedad y los retos que enfrenta esta profesión, se identificaron oportunidades de renovación para los programas de contaduría pública en tres dimensiones: disciplinar, metodológica e institucional; que se desarrollan a continuación.

\section{Oportunidades para el desarrollo de los programas de contaduría pública: disciplinar}

Las oportunidades para alcanzar una educación profesional competente en contaduría pública, de impacto regional, con proyección global, que aporte en la gestión integral de las organizaciones, sustentada en la ética y la responsabilidad social y ambiental, requieren el desarrollo de los siguientes puntos.

\section{Contaduría pública de impacto regional}

El desarrollo económico y social del país se orienta en múltiples dimensiones que dan origen a la diversidad de la actividad económica en sus diferentes sectores. Las organizaciones se constituyen en el centro del desarrollo económico y bienestar social del país; organizaciones que pueden ser públicas o privadas, con o sin ánimo de lucro, grandes, medianas o pequeñas, sociedades o personas naturales, de todos los sectores de la economía, bien sea industrial, comercial, servicios, agropecuario, construcción, servicios financieros, que en el actual contexto de desarrollo del país enfrentan los retos de la competencia local y global. Ante este contexto diversificado se hace evidente el papel protagónico que deben asumir los profesionales de la contaduría pública, sensibles respecto de las necesidades particulares que se generan desde sus regiones, para que mediante su apoyo puedan aportar significativamente al desarrollo de las organizaciones, sus comunidades y a su propio su desarrollo profesional. 
De esta manera, los nuevos programas de contaduría pública van a aportar a la excelencia de la educación superior en Colombia en el escenario de la paz, para que "todas las regiones cuenten con educación superior" de calidad.

\section{Contaduría pública con proyección global}

Dados los actuales esquemas de mercados altamente globalizados que implican desarrollo comercial y económico abierto en el ámbito mundial, los programas de contaduría pública deben ser propuestos a partir de las Normas Internacionales de Información Financiera y de Aseguramiento de la Información, como aporte para que las organizaciones avancen hacia el logro de mayores estándares de productividad y competitividad.

En este escenario, la respuesta se orienta a consolidar programas de contaduría pública con enfoque global. Los cuales, además de implementar en todos sus cursos disciplinares los estándares internacionales para la gestión de la información financiera y de aseguramiento de la información, deben proponer cursos para fortalecer los conocimientos que se requieren para enfrentar el actual contexto globalizado de los negocios en la dinámica económica mundial.

Tal como lo señalan Arum y Van de Water, Harai, Klasek, Mestenhauser, Green y Olson, Powell, Green y Shoenberg, Delgado (citados en Ortiz, 2015) la internacionalización en los programas de estudio debe ser desarrollada desde diferentes actividades para fomentar una dimensión intercultural, desde el currículo, la movilidad académica y la cooperación. Lo anterior con el objetivo de aportar al logro de habilidades, conocimientos, actitudes y valores que permitan a los futuros profesionales enfrentar el actual contexto altamente globalizado. 


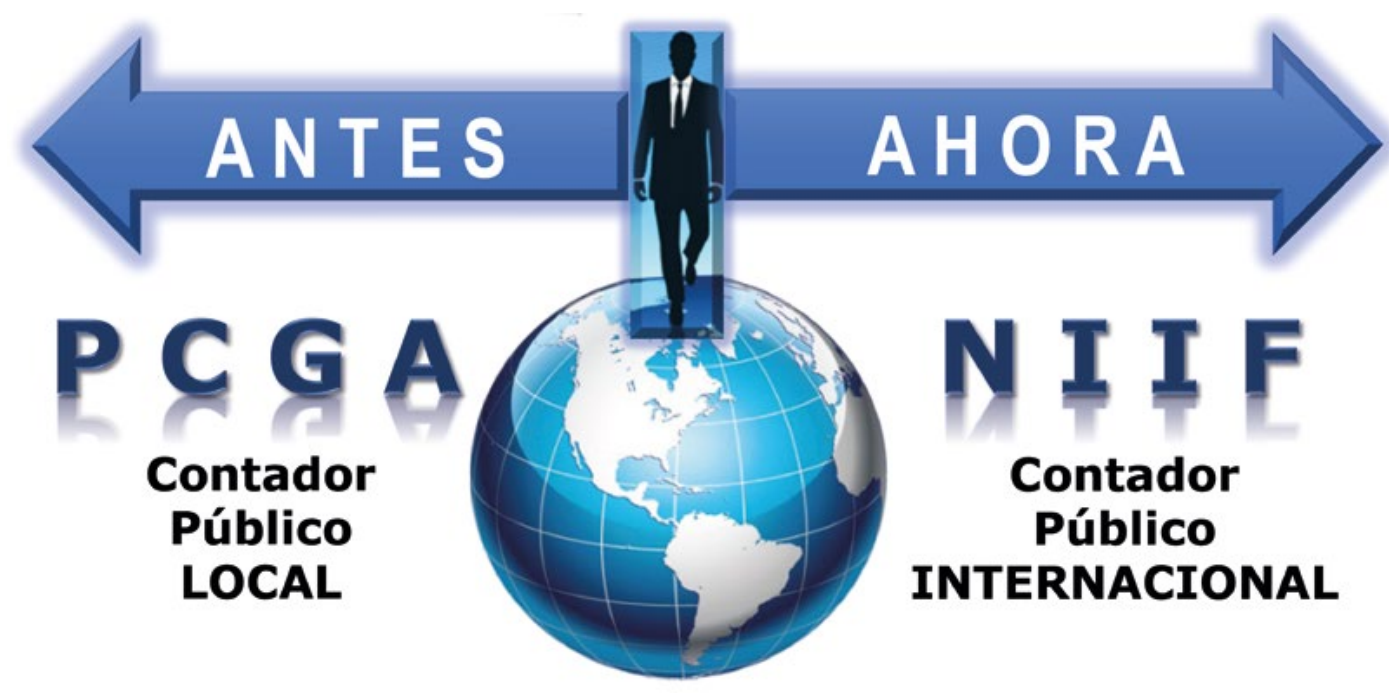

Figura 20. Oportunidad para el desempeño profesional en el contexto globalizado.

Fuente: elaboración propia a partir del Informe CONFECÁMARAS 2017

Conerando la actual política pública, establecida a partir de la Ley 1314 de 2009, es necesario avanzar en la formación de los profesionales de la contaduría pública basada en los actuales marcos conceptuales propuestos bajo estándares internacionales para la gestión de la información financiera y del aseguramiento de la información, que deben incorporar las organizaciones del país en la obtención de su información financiera.

En este contexto, como lo señalan Muñoz (2014), Villarreal, Martínez y Muñoz (2016), y Rodríguez (2017), se requiere que los diseños curriculares de los programas de contaduría pública del país sean actualizados para su desarrollo con base en los nuevos marcos conceptuales. Esto ocasiona un cambio de enfoque respecto de la educación del contador público, se pasa de un contexto local con los principios de contabilidad generalmente aceptados en Colombia, a un contexto internacional con la aplicación de las normas internacionales de información financiera y de aseguramiento de la información. 
En este desarrollo, es necesario aportar a los futuros profesionales de la contaduría pública, además de la titulación profesional como contador público, la certificación como contador internacional. Para ello es necesario aportar en la preparación de la comunidad estudiantil de los programas de contaduría pública para que avancen en la obtención de esta certificación ante los dos organismos internacionales reconocidos para esta función, a saber, Association of Chartered Certified Accountants (AcCA), y el Institute of Chartered Accountants in England and Wales (ICAEW).

La certificación Internacional en IfRS, Full y Pymes, con AccA como organismo mundial líder en contabilidad tiene una trayectoria que supera los 110 años de permanencia y oficinas en 92 países del mundo. La certificación Acca es la más importante y de mayor crecimiento en el mundo que ha desarrollado una red global de miembros, estudiantes, socios de educación y empleadores, con 178.000 miembros y más de 455.000 estudiantes, ubicados en 181 países.

La certificación Internacional en IFRS, Full y Pymes, con ICAEW, como organización profesional líder en el mundo que promueve, desarrolla y apoya a más de 146.000 contadores públicos en más de 160 países, con oficinas en Londres, Beijing, Bruselas, Dubai, Hong Kong, Kuala Lumpur y Singapur; y a través de Partners in Learning en todo el mundo, ofrece esta certificación internacional para proteger la calidad e integridad de la profesión contable.

Para avanzar en la formación integral del contador público y su desempeño competente en el actual contexto globalizado, es necesario considerar el desarrollo de competencias de comunicación en segundo idioma y, como lo señalan Vargas, Tejada y Colmenares (2011), mejorar en el desarrollo de competencias en inglés, necesario en los currículos de los programas de contaduría pública.

El dominio de un segundo idioma aporta a potenciar la comunicación en el contexto internacional. Por ello, los programas de contaduría pública deben avanzar en sus propuestas que coadyuve a los futuros profesionales a alcanzar el nivel B2 en inglés, según el Marco Común Europeo de Referencia para las Lenguas (MCERL). 
Lograr el nivel B2 en inglés permite que los estudiantes desarrollen competencias para entender las ideas principales de textos complejos que traten temas de carácter técnico, relacionarse con hablantes nativos con un grado suficiente de fluidez y naturalidad, y producir textos claros y detallados sobre temas diversos, así como defender un punto de vista sobre temas generales indicando las ventajas y desventajas de las distintas opciones que enfrentan.

De esta manera se orientan los programas de contaduría pública hacia la formación del contador público internacional, para la excelencia de la educación superior, en coherencia con el Acuerdo por lo Superior 2034, una educación superior de calidad con enfoque internacional.

\section{Contaduría pública para la gestión integral en las organizaciones}

Los profesionales de la contaduría pública son vistos como agentes de información, certificación y fiscalización, como generadores y gestores de la información financiera y de su aseguramiento. Por ello les corresponde la responsabilidad de analizar dicha información y aportar en el proceso de toma de decisiones para la gestión de las organizaciones. Así, es necesario que el contador conozca integralmente lo que es una organización para potenciar su actuar profesional en una dimensión superior que redunde en beneficios para el crecimiento y fortalecimiento empresarial.

De esta manera, se potencia la oportunidad de desempeño profesional al considerar una formación integral, que aporte significativamente en la gestión de las organizaciones y propenda por la educación superior reconocida por su calidad.

\section{Contaduría pública con fundamento ético y socialmente responsable}

La contabilidad históricamente se ha leído en una constante relación con las dinámicas de la vida cotidiana. Uno de los grandes hitos está en la revolución 
científica y tecnológica que la hizo base fundamental para el desarrollo de las aspiraciones de la sociedad, delegándole, por consiguiente, una función de espíritu moral (Sunder, 2005). Ello, al ser garante de la confianza de la relación entre terceros y al vincular las poblaciones desde las regiones al aparato productivo como materias primas, así sienta los pilares de una nueva ética, la cual estará orientada a la sostenibilidad y el bienestar de las organizaciones, las comunidades y su medio ambiente.

Para ello, los programas de contaduría pública se deben orientar al desarrollo de bases importantes sobre la conciencia ética del ser humano y del ser profesional para enfrentar la responsabilidad que les corresponde frente a las comunidades, las organizaciones y el medio ambiente que éstas impactan. Además, los programas de contaduría pública deben considerar vincular creativa y articuladamente la investigación desde su ejercicio profesional, que permita identificar problemáticas y proponer soluciones que aporten significativamente al desarrollo económico, social y humano sostenible de las comunidades locales, regionales y globales con calidad, eficiencia y equidad social.

\section{Oportunidades para el desarrollo de los programas de contaduría pública: modalidad virtual}

Los desarrollos tecnológicos han permitido la interacción a través de entornos virtuales de aprendizaje, aportan a la educación para toda la comunidad del país, en todo momento y con cobertura en todas las regiones, y fomentan el aprendizaje autónomo con calidad, eficiencia y equidad social. Apoyado en e-Learning se promueve la construcción de procesos permanentes de aprendizaje significativo, colaborativo y autónomo. Este debe ser considerado desde las instituciones de educación superior en la formación de los profesionales para el aporte al mejoramiento de la calidad de vida de la comunidad y al impacto educativo en todas las regiones del país, incluso aquellas más apartadas donde la educación tradicional no hace presencia. 
Como lo señala García (2005), quien se refiere al aporte del e-Learning al sistema educativo:

El sector educativo ha encontrado en esta tecnología un excelente medio para romper con las limitantes geográficas y temporales que los esquemas tradicionales de enseñanza-aprendizaje conllevan, revolucionando, y cambiando a la vez, el concepto de educación a distancia. Su adopción y uso han sido amplios, lo que ha permitido un desarrollo rápido y consistente en el que la Web ha ido tomando distintas formas dentro de los procesos educativos. (p.1).

De acuerdo el Proyecto Académico Pedagógico Solidario propuesto por la UNAD (2011), el e-Learning se constituye en un ambiente de aprendizaje electrónico con canales múltiples de comunicación y altamente interactivo, dinámico y creativo. Permite a los estudiantes apropiarse, comprender y transferir el conocimiento con el apoyo de las TIC, de una manera flexible que fomenta la interacción e interrelación entre el e-currículo, e-medios, e-mediadores, e-mediaciones, e-evaluación, la e-comunidad, el e-bienestar, la e-calidad y la e-investigación, en los ambientes virtuales de aprendizaje AVA.

Para ello, el campus virtual de la UNAD es un referente de institución pública líder en educación virtual en Colombia, que permite el desarrollo de relaciones para el aprendizaje virtual. El sitio web de acceso único para los servicios de la comunidad académica está conformado por un conjunto de subplataformas que estandarizan los procesos académicos y administrativos de la institución. Las subplataformas que conforman el Campus Virtual son: Contents -o Laboratorio pedagógico de contenidos-, Mis Cursos Virtuales -donde el estudiante realizan su proceso de aprendizaje-, Registro y Control Académico - bases de datos-, Formador de Formadores - o Plataforma de Formación de Formadores-, la biblioteca virtual, así como los servicios conexos de Radio UNAD Virtual y TV a través Internet.

En la UNAD, los Ambientes Virtuales de Aprendizaje (AVA) permiten el desarrollo de cursos a través de entornos de aprendizaje: información inicial, 
conocimiento, aprendizaje colaborativo, aprendizaje práctico, seguimiento y evaluación y gestión. Dichos entornos no sólo permiten organizar la información, sino que operan como el escenario en el cual se dinamizan las intencionalidades didácticas y pedagógicas que propenden por fortalecer y promover procesos de aprendizaje significativo, colaborativo y autónomo. En cada uno de los entornos virtuales de aprendizaje se encuentran recursos que permiten el desarrollo del trabajo independiente y colaborativo, y brindan al estudiante la posibilidad de trazar rutas de acción para su proceso formativo (PAP Solidario UNAD, 2011)

De acuerdo con las necesidades de la población en educación contable, los programas de contaduría pública propuestos a partir de la utilización intensiva de la tecnología, apoyados en e-learning, aportan en el avance de la educación superior universal y de calidad en todo tiempo y lugar, de forma inclusiva, con acceso, permanencia y graduación para todos.

\section{Oportunidades para el desarrollo de los programas de contaduría pública: flexibilidad}

La formación profesional de contadores públicos debe responder a las necesidades propias del campo de conocimiento contable, las políticas públicas y las tendencias internacionales. Además, debe ser pertinentemente para responder a las necesidades del sector empresarial y de la población del país, por ello, es necesario avanzar en la propuesta de currículos flexibles; donde la "flexibilidad" sea concebida en diferentes orientaciones. Esto va a permitir fortalecer la propuesta académica para el logro de profesionales de la contaduría pública competentes, que respondan a las necesidades de la profesión, el sector empresarial y la comunidad.

La flexibilidad curricular se apreciará en los diferentes campos de formación: básica, sociohumanística, integral y disciplinar, que conforman el currículo y 
aportan en la formación integral del futuro profesional. Esto es apreciable tanto en cursos obligatorios y electivos, como en la oportunidad de inter, multi y transdisciplinariedad que propicie la interacción entre pares del programa, de otros programas afines y no afines al campo del conocimiento contable.

En otra dimensión, la flexibilidad se aprecia en la distribución de cursos electivos, los cuales deben traspasar su presencia en el campo disciplinar y trascender a los demás campos del conocimiento, que potencie la formación profesional del contador público. Esta dimensión brinda oportunidades a la comunidad del país para elegir cómo terminar su proceso formativo, de acuerdo con su proyecto de vida, las necesidades del contexto regional y desarrollo profesional, que aporte al impacto social en las diversas comunidades del país.

Finalmente, se debe señalar la importancia, para los programas de contaduría pública, de aplicar a los procesos de matrícula y derechos pecuniarios el sistema de créditos académicos. Esta flexibilidad aporta a la comunidad estudiantil la decisión del número de créditos a cursar, en consideración de sus propias necesidades de acuerdo con su disposición de tiempo y situación económica.

Con las consideraciones planteadas, se estará contribuyendo en la formación de profesionales de la contaduría pública capaces de actuar en el actual contexto globalizado, con sólidos conocimientos disciplinares, formación integral, socialmente responsables y elevados valores éticos. Ello mediante el desarrollo del pensamiento crítico, la acción investigativa, la proyección social y la utilización intensiva de las tecnologías de la información y las comunicaciones para el aporte al desarrollo económico, social y del medio ambiente de las organizaciones y de su entorno, con pertinencia regional y proyección global. 


\section{Oportunidad para articular la investigación y la proyección social, para el desempeño profesional ético y socialmente responsable}

Como motor dinamizador, la investigación se constituye en base fundamental que posibilita la identificación de problemáticas o necesidades latentes propias de comunidades sociales y empresariales desde los contextos regionales donde hacen presencia. Permite avanzar en el desarrollo de relaciones solidarias que promueven la inclusión, la equidad social y contribuyen a la preservación ambiental mediante el aporte de soluciones a los problemas locales, regionales y nacionales tendientes al desarrollo armónico, sostenible y perdurable de las organizaciones en todas las zonas y regiones del país.

Este desarrollo debe ser asumido desde las propuestas de los programas de contaduría pública, para alcanzar la conciencia sobre la responsabilidad que le es implícita a este tipo de profesionales, desde su función ética y social. A partir de esta conciencia, apoyar la generación de impacto regional acorde con las necesidades de las comunidades locales en las diferentes regiones del país.

Para ello, la articulación de la investigación y la proyección social con fundamento ético y socialmente responsable se constituyen en estrategia dinamizadora que permite el desarrollo y la diversidad empresarial, social, económica propias de cada región. Aspectos que permiten el desarrollo de la conciencia critico-social y conciencia ética en el futuro profesional de la contaduría.

Con este propósito, se deben identificar las problemáticas que afectan las comunidades, las organizaciones y el medio ambiente en las diferentes regiones, a partir de las cuales - y mediante la investigación como motor que coadyuve en la identificación de soluciones- se pueda avanzar en el desarrollo económico, social y humano, sostenible de las comunidades y las organizaciones, desde los diferentes contextos regionales del país. 


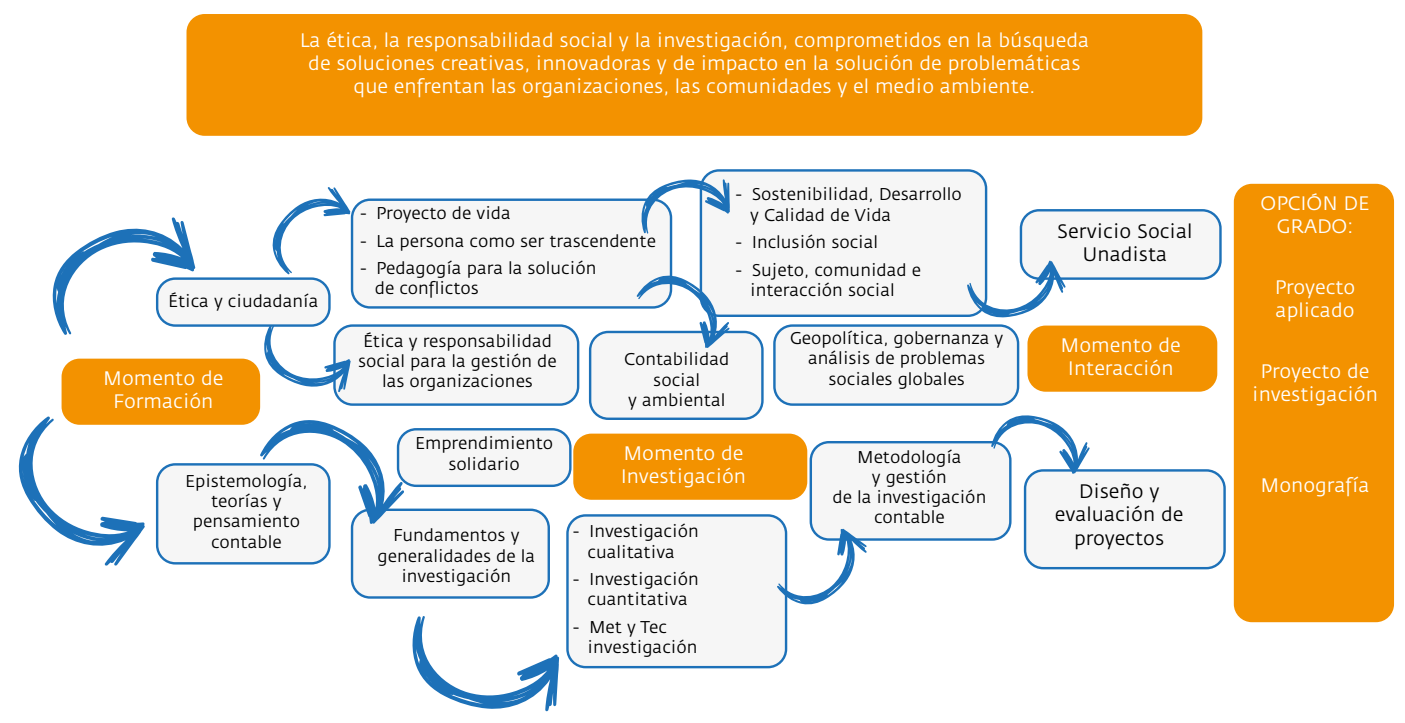

Figura 21. Articulación de la investigación y la proyección social.

Fuente: elaboración propia

Para ello, se debe concebir en los currículos la investigación como eje articulador y generador de impacto social. Mediante su articulación con la proyección social y la formación integral y ética dinamizan el avance en el logro los programas tanto para la comunidad como para las organizaciones.

\section{Oportunidad potenciales y existentes para el desempeño de los profesionales}

De acuerdo con la información disponible en el Observatorio Laboral para la Educación (2017), se analiza el perfil académico y condiciones de empleabilidad para los graduados de programas de educación superior. Este muestra que, desde 1960 hasta 2014, se han otorgado en el país un total de 4.738 .167 títulos de educación superior en todos los niveles de formación, desde el técnico profesional hasta el doctorado, de los cuales 3.179.102 equivalentes al 67,1 \% fueron entregados a partir de la década del 2000. 
Como se aprecia en la figura 22, los programas de formación de nivel profesional son los que mayor demanda tienen y, adicionalmente, muestran una tendencia creciente al pasar del 44,3\% en 2011 a 46,3\% en el año 2014.

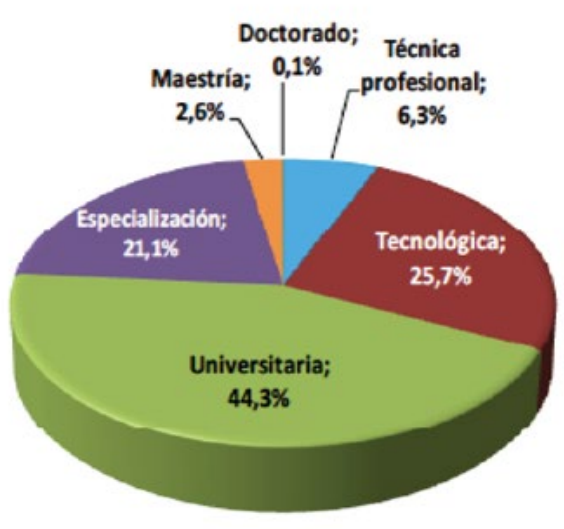

2011

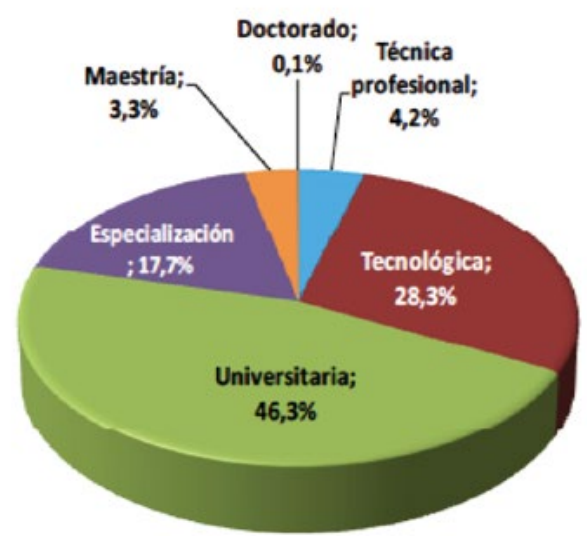

2014

Figura 22. Titulaciones de educación superior por nivel de formación, 2011 y 2014 Fuente: MEN, Observatorio Laboral para la Educación, 2017

En el año 2014, en Colombia se otorgaron 358.910 títulos de educación superior, frente a 299.816 en 2011 representando incremento del 19,7\%. Respecto de las áreas de conocimiento, las carreras con más títulos otorgados fueron: economía, administración, contaduría y afines, 31,7 \% en 2011 y 37,0 \% en 2014; ingeniería, arquitectura, urbanismo y afines (22,3\% y 23,4 \%); ciencias sociales y humanas (16,9\%) y 15,3\%); ciencias de la educación (13,1\% y $9,9 \%)$.

En la figura 23 se presenta la información de los graduados del período 2011 a 2014 en Bogotá, por corresponder a la ciudad que concentra la mayor participación de graduados del país, muy por encima del resto de regiones, cuya tendencia ilustra las preferencias por los programas de educación en el resto del país. Como se aprecia, del 1.117.912 graduados, el $51 \%$, es decir 567.288 graduados, corresponden al nivel universitario y respecto del área de formación en economía, administración, contaduría y afines se encuentran 361.779 
graduados, que representan el $64 \%$ de los egresados de nivel universitario, evidenciándose una preferencia por estos programas universitarios entre los cuales se encuentra el programa de contaduría pública.

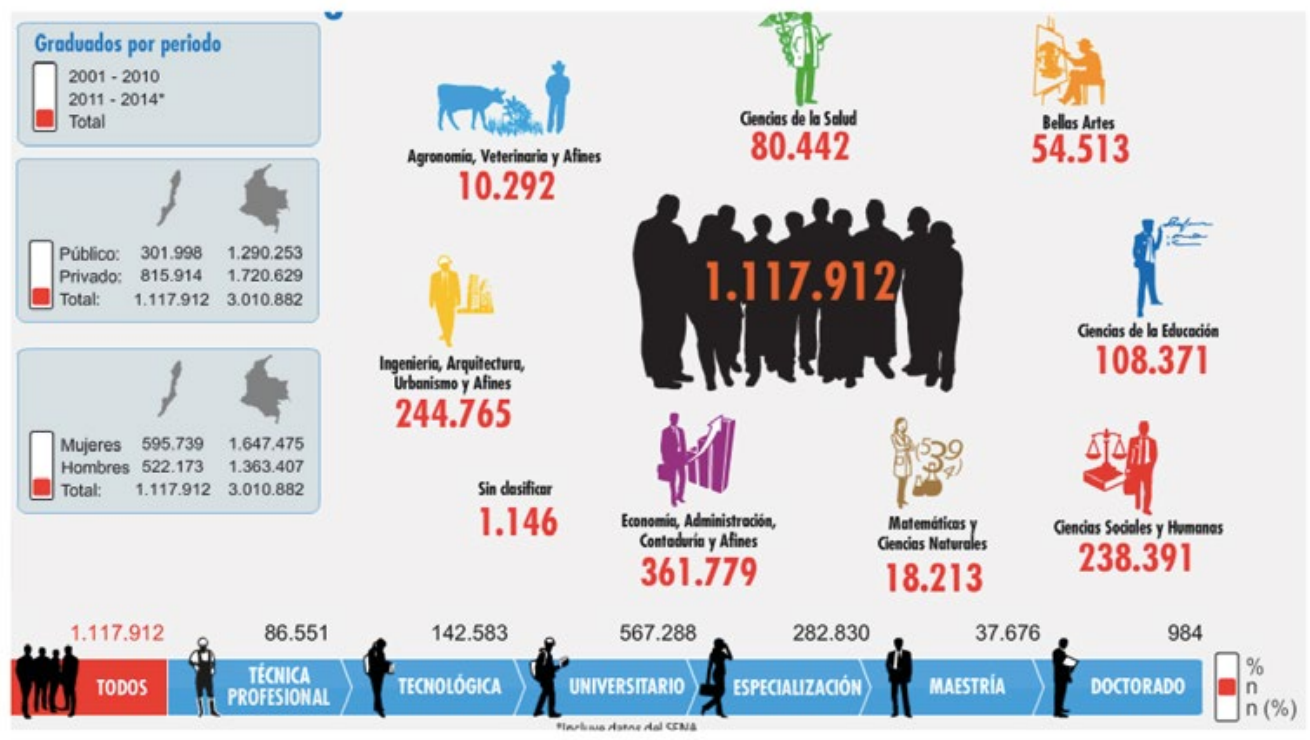

Figura 23: Graduados en educación superior a 2014.

Fuente: MEN, Observatorio Laboral para la Educación, 2017

En el ámbito nacional, se aprecia cómo los programas de educación superior impactan unas regiones en mayor proporción que otras. Esto se evidencia en la concentración e impacto, principalmente, en la capital del país y luego en ciudades como Medellín, Cali y Santander. Impacto que contrasta con las demás regiones de Colombia, tal como se ilustra en la siguiente gráfica. 


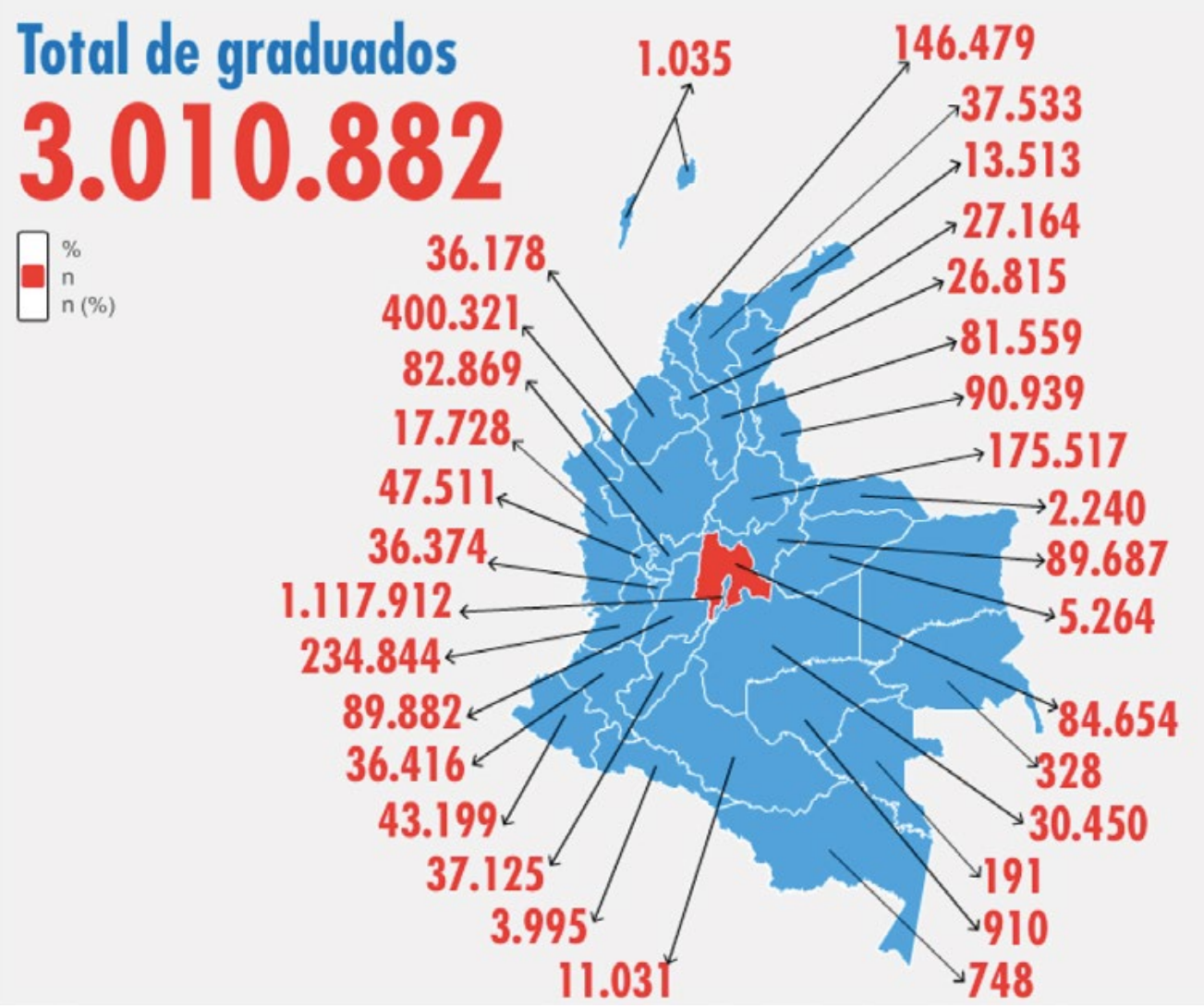

$\begin{array}{lll}\checkmark \text { Amazonas } & \checkmark \text { Cesar } & \checkmark \text { Norte de Santander } \\ \checkmark \text { Antioquia } & \checkmark \text { Chocó } & \checkmark \text { Putumayo } \\ \checkmark \text { Arauca } & \checkmark \text { Córdoba } & \checkmark \text { Cuindio } \\ \checkmark \text { Atlántico } & \checkmark \text { Cundinamarca } & \checkmark \text { Risaralda } \\ \checkmark \text { Bogotá } & \checkmark \text { Guainía } & \checkmark \text { San Andrés } \\ \checkmark \text { Bolivar } & \checkmark \text { Guajira } & \checkmark \text { Santander } \\ \checkmark \text { Boyacá } & \checkmark \text { Guaviare } & \checkmark \text { Sucre } \\ \checkmark \text { Caquetá } & \checkmark \text { Huila } & \checkmark \text { Tolima } \\ \checkmark \text { Caldas } & \checkmark \text { Magdalena } & \checkmark \text { Valle del Cauca } \\ \checkmark \text { Casanare } & \checkmark \text { Meta } & \checkmark \text { Vaupés } \\ \checkmark \text { Cauca } & \checkmark \text { Nariño } & \checkmark \text { Vichada }\end{array}$

Figura 24. Graduados en Colombia por regiones.

Fuente: MEN, Observatorio Laboral para la Educación, 2017 
Respecto del programa de contaduría pública, para el período comprendido entre los años 2000 a 2014, se evidencia que es el segundo programa de mayor demanda en Colombia.

Deacuerdoconla información disponibleen el Observatorio Laboral Colombiano, que consolida la información de los egresados de programas de Contaduría pública del año 2001 al año 2015, se aprecia un incremento del $135 \%$ en el número de egresados del año 2001, con 7.037 egresados respecto al año 2015 y con 16.533 egresados. Esto indica que la contaduría pública es una profesión de gran interés para la población colombiana que desea obtener su titulación profesional.

Tabla 10. Títulos otorgados en contaduría pública 2001 a 2015.

\begin{tabular}{|c|c|c|c|c|c|c|c|c|c|c|c|c|c|c|c|c|}
\hline & \multicolumn{16}{|c|}{ PERIODO GRADUACIÓN } \\
\hline & $\nabla$ & $\nabla$ & $\nabla$ & 7 & $\boldsymbol{\nabla}$ & $\boldsymbol{F}$ & 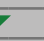 & $\nabla$ & $\nabla$ & $\boldsymbol{\nabla}$ & $\nabla$ & $\boldsymbol{F}$ & $\boldsymbol{V}$ & $\boldsymbol{\nabla}$ & $\boldsymbol{\nabla}$ & \\
\hline & $\overline{\text { ¿্র }}$ & \&్ & ర్ & $\underset{\sim}{+}$ & $\stackrel{\text { \& }}{\circ}$ & \&ి & $\overline{\overbrace{}^{2}}$ & \&̊ & ठ̊ & 은 & $\overline{\bar{\alpha}}$ & กั & $\bar{n}^{m}$ & $\stackrel{\text { a }}{\circ}$ & 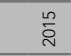 & 㶾 \\
\hline Contaduría pública & 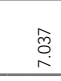 & 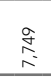 & $\underset{\substack{\infty \\
\hdashline}}{\stackrel{a}{\sim}}$ & $\begin{array}{l}\overline{8} \\
\infty \\
\infty\end{array}$ & $\begin{array}{c}m \\
\substack{\infty \\
0 \\
0} \\
\end{array}$ & 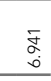 & 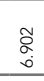 & $\underset{\substack{1 \\
\infty \\
\infty}}{\circ}$ & $\underset{\infty}{\infty}$ & $\stackrel{\infty}{\sim} \underset{\sim}{\sim}$ & 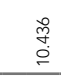 & 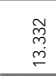 & 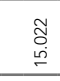 & 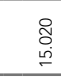 & 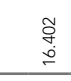 & $\begin{array}{l}\stackrel{\infty}{\infty} \\
\stackrel{\vec{J}}{-}\end{array}$ \\
\hline $\begin{array}{l}\text { Contaduría pública con énfasis en sistemas } \\
\text { y economía solidaria }\end{array}$ & - & - & - & - & - & - & 14 & 17 & - & - & - & 12 & - & - & - & 43 \\
\hline $\begin{array}{l}\text { Contaduría pública énfasis en sistemas } \\
\text { y economía solidaria }\end{array}$ & - & - & - & 30 & 35 & 69 & 35 & 20 & 13 & - & - & - & - & - & - & 202 \\
\hline Contaduría pública y finanzas internacionales & - & - & - & 3 & 7 & 11 & 8 & 8 & 10 & 22 & 24 & 33 & 18 & 32 & 23 & 199 \\
\hline Profesional en contaduría pública & - & - & - & - & & & & & 6 & 31 & 59 & 81 & 96 & 119 & 108 & 500 \\
\hline Total & $\stackrel{\substack{o \\
i}}{n}$ & 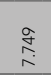 & 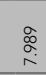 & $\begin{array}{ll}0 \\
0 \\
\infty \\
\infty \\
\infty \\
\infty\end{array}$ & 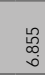 & 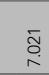 & 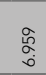 & 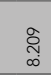 & $\underset{\infty}{\infty}$ & হ్̀ & 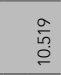 & 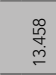 & 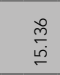 & 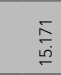 & 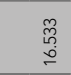 & $\begin{array}{l}\stackrel{a}{a} \\
\text { oj }\end{array}$ \\
\hline
\end{tabular}

Fuente: MEN, Observatorio Laboral para la Educación, 2017

El nivel de salarios de los profesionales de la contaduría pública varía desde su momento de titulación y en la medida que van adquiriendo experiencia después de su graduación. Se aprecia una tendencia interesante del incremento del nivel salarial para un contador público que cuente con experiencia respecto del recién egresado. Según los datos del Observatorio Laboral, actualizados al año 2015, para un graduado en el año 2010 que tenga experiencia laboral de cinco años al 2015, el ingreso laboral fluctúa entre \$2.014.008 y \$2.133.186. En comparación, el profesional recién egresado tiene ingresos que van desde $\$ 1.351 .362$ y hasta $\$ 1.618 .486$. En ambos casos se aprecia que el contador público en finanzas internacionales es el de mejor ingreso salarial (tabla 11). 
Tabla 11. Vinculación laboral general. Programa contaduría pública

\begin{tabular}{llll} 
Programa formación académica & $\begin{array}{l}\text { Graduados } \\
\text { año } 2015\end{array}$ & $\begin{array}{l}\text { Graduados } \\
\text { año 2014 }\end{array}$ & $\begin{array}{l}\text { Gradua- } \\
\text { dos } \\
\text { año } 2010\end{array}$ \\
\hline Contaduría pública & $\$ 1.351 .362$ & $\$ 1.470 .481$ & $\$ 2.014 .008$ \\
\hline Contaduría pública y finanzas internacionales & $\$ 1.618 .486$ & 1.711 .469 & $\$ 4.322 .857$ \\
\hline Profesional en contaduría pública & $\$ 1.404 .671$ & 1.545 .595 & $\$ 2.133 .186$ \\
\hline
\end{tabular}

Fuente: MEN, Observatorio Laboral para la Educación, 2017.

El momento presente es coyuntural para la contaduría pública por el proceso de convergencia a estándares internacionales para la información financiera y el aseguramiento de la información. Este proceso presenta un gran avance y desarrollo a nivel mundial, y ha llevado a colocar esta profesión como una de las más apetecidas tanto en el ámbito nacional como internacional.

\section{Conclusiones}

La articulación entre investigación y proyección social permiten coadyuvar al desempeño ético y socialmente responsable de los futuros profesionales y contribuye al impacto regional pertinente para avanzar en la formación integral del futuro profesional.

La formación integral se orienta desde la dimensión ética del ser como individuo en sociedad y la ética como profesional de la contaduría pública. El contador público es un generador de confianza, consciente de la responsabilidad social que le es implícita, con la proyección social y la investigación formativa, con responsabilidad social y ética.

Considerar la relevancia de la internacionalización del currículo en las propuestas académicas de los programas de contaduría pública, como elementos asociados para la internacionalización del currículo, el acogimiento de los estándares internacionales para la educación contable, la certificación profesional internacional, el desarrollo de competencias en segundo idioma, movilidad internacional, entre otros; se constituye en un aporte valioso en el 
proceso de desarrollo de competencias orientadas al logro del desempeño profesional de la contaduría pública en un contexto globalizado y cambiante. Este aportará a los futuros profesionales los conocimientos, cualidades y capacidades para comunicarse, actuar y tomar decisiones, de acuerdo con el contexto real en el que se desempeñan las organizaciones.

\section{Referencias}

Association of Chartered Certified Accountants (AcCA). (2017). Certificación Internacional en IFRS, Full y Pymes. Recuperado de: https://www.accaglobal.com/gb/en.html

Consejo Nacional de Educación Superior (CESU). (2014). Acuerdo por lo superior 2034.

Recuperado de: https://www.dialogoeducacionsuperior.edu.co/1750/articles-321515_ recurso_1.pdf

Congreso de la República de Colombia. (2009). Ley 1314 de julio 13 de 2009.

Recuperado de: http://www.secretariasenado.gov.co/senado/basedoc/ ley_1314_2009.html

Congreso de la República de Colombia. (2015). Ley 1753 de junio 9 de 2015, Plan Nacional de Desarrollo 2014-2018 "Todos por un nuevo país: Paz, equidad, educación". Recuperado de: http://www.sic.gov.co/sites/default/files/documentos/ LEY-1753-15\%20Plan\%20Nacional\%20de\%20Desarrollo\%202014\%20-\%202018.pdf

García, F. (2005). Estado actual de los sistemas e-learning. Teoría de la Educación. Educación y Cultura en la Sociedad de la Información, 6(2).

Institute of Chartered Accountants in England and Wales (ICAEW). (2017). Certificación Internacional en IFRS, Full y Pymes. Recuperado de: https://www.icaew.com 
Ministerio de Educación, Cultura y Deporte. (2002). Marco Común Europeo de Referencia para las lenguas: enseñanza, aprendizaje, evaluación. Recuperado de: https://www.mineducacion.gov.co/1621/articles-237704_archivo_pdf_marco_ europeo.pdf

Ministerio de Educación Nacional. (s.f.) Colombia, la más educada en el 2025. Recuperado de: https://www.mineducacion.gov.co/1621/articles-355154_foto_ portada.pdf

Muñoz, C. (2014). Estándares internacionales de educación en contabilidad y aseguramiento: Nuevos retos de la profesión contable. Tendencias, 15(2), 118-135.

Ortiz, A. (2012). Internacionalización del Currículo. Mundo CERI, 2, 4-11.

Presidencia de la República de Colombia. (1993). Decreto 2649 de diciembre 29 de 1993. Recuperado de: https://bibliotecadigital.ccb.org.co/bitstream/handle/11520/13812/ Decreto\%202649\%20de\%201993.pdf?sequence=1\&isAllowed=y

Rodríguez, E. (2017). La estandarización en el currículo educativo: la punta del iceberg de la homogeneización. Alteridad. Revista de Educación, 12(2), 248-258.

Sunder, S. (2005). Teoría de la Contabilidad y el Control. Facultad de Ciencias Económicas. Colombia: Universidad Nacional de Colombia. Unibiblos.

Universidad Nacional Abierta y a Distancia, UNAD. (2011). Proyecto Académico Pedagógico Solidario, PAP Solidario. Recuperado de: https://academia.unad.edu. co/images/pap-solidario/PAP\%20solidario\%20v3.pdf

Vargas, A.; Tejada, H. y Colmenares, S. (2011). Estándares básicos de competencias en lenguas extranjeras (inglés): una lectura crítica. Lenguaje, [S.I.], 36(1).

Villarreal, J.: Martínez, J. y Muñoz, C. (2016). De la educación contable internacional al desarrollo de competencias. Revista ESPACIOS, 37 (33). 
